\title{
Phase space geometry in scalar-tensor cosmology
}

\author{
Valerio Faraoni \\ Physics Department, Bishop's University \\ Lennoxville, Quèbec, Canada J1M 1Z7 \\ and \\ Physics Department, University of Northern British Columbia \\ 3333 University Way, Prince George, B.C., Canada V2N $4 Z 9$ \\ emailvvfaraoni@unbc.ca
}

\begin{abstract}
We study the phase space of spatially homogeneous and isotropic cosmology in general scalar-tensor theories. A reduction to a two-dimensional phase space is performed when possible - in these situations the phase space is usually a twodimensional curved surface embedded in a three-dimensional space and composed of two sheets attached to each other, possibly with complicated topology. The results obtained are independent of the choice of the coupling function of the theory and, in certain situations, also of the potential.
\end{abstract}




\section{Introduction}

Brans-Dicke and scalar-tensor gravity have been studied for over four decades. The original motivation for the introduction of Brans-Dicke theory [1] came from Mach's principle and cosmology but since Brans and Dicke's original paper, significant motivation has been added for research in scalar-tensor gravity. First, Brans-Dicke theory has been generalized to scalar-tensor theories with coupling functions instead of coupling constants [2]. More important, it has been realized that scalar-tensor gravity contains features that are common to supergravity and string or M-theory. The low-energy limit of the bosonic string theory reduces to a Brans-Dicke theory with Brans-Dicke parameter $\omega=-1[3]$.

Renewed interest in cosmology in scalar-tensor gravity (see Refs. [4, 5]) has been motivated by the extended [6] and hyperextended [7] scenarios of inflation in the early universe and by scalar-tensor models of dark energy trying to explain the present acceleration of the universe [8]. Even with the simplifying symmetry of spatially homogeneous and isotropic Friedmann-Lemaitre-Robertson-Walker (hereafter "FLRW") cosmology, the field equations of scalar-tensor gravity for the metric and the Brans-Dicke-like scalar field are complicated, only a few exact solutions are available, and a phase space picture is very useful for the qualitative description of the dynamics. A few excellent and detailed works on the dynamics of Brans-Dicke homogeneous and isotropic cosmological models are available [9]-[11]. The possibilities studied in the literature include giving a mass or other potential $V(\phi)$ to the Brans-Dicke-like field $\phi$ (as is natural in particle physics), adding a perfect fluid while keeping $V \equiv 0$, adding a cosmological constant $\Lambda$, and/or considering FLRW spaces with the three possible values $0, \pm 1$ of the curvature index $K$. However, the geometric structure of the phase space, including its dimensionality, is still poorly understood for scalar-tensor theories more general than Brans-Dicke theory. In addition, the studies of the dynamics available in the literature often use variables that mix the scale factor $a(t)$ of the FLRW metric with the Brans-Dicke-like field $\phi$. Although convenient from the formal point of view, the use of these variables tends to obscure the physical picture and it may be difficult to rephrase results in terms of the physical variables traditionally used in cosmology, such as the scale factor $a$, the Hubble parameter $H$, the comoving time $t$, and the scalar field $\phi$.

In this paper we focus on spatially homogeneous and isotropic FLRW spaces described by the metric

$$
d s^{2}=-d t^{2}+a^{2}(t)\left[\frac{d r^{2}}{1-K r^{2}}+r^{2}\left(d \theta^{2}+\sin ^{2} \theta d \varphi^{2}\right)\right]
$$

in comoving coordinates $(t, r, \theta, \varphi)$ and with curvature index $K$. We consider scalar- 
tensor theories of gravity described by the action

$$
S^{(S T)}=\frac{1}{16 \pi} \int d^{4} x \sqrt{-g}\left[\phi R-\frac{\omega(\phi)}{\phi} g^{a b} \nabla_{a} \phi \nabla_{b} \phi-V(\phi)\right]+S^{(m)}
$$

where $S^{(m)}$ is the action describing ordinary matter, $g$ is the determinant of the metric tensor $g_{a b}, \nabla_{c}$ is the covariant derivative operator associated with $g_{a b}, R$ is the Ricci curvature of spacetime, and $\phi$ is the gravitational Brans-Dicke-like scalar self-interacting through the potential $V(\phi)$. The metric signature is,,,,$-+++ \square \equiv g^{b c} \nabla_{b} \nabla_{c}$, and we follow the notations and conventions of Ref. [12].

The study of FLRW cosmology at very early times has limited value because isotropization is probably achieved by inflation, which washes away information about the preinflationary state of the universe. At very early times before inflation the universe may have been anisotropic (see Refs. [13] for studies of isotropization of Bianchi models in scalar-tensor gravity). However, it is meaningful to consider FLRW universes during and after inflation and in particular during the present and future evolution of the universe. In the quest for successful models of dark energy able to explain the present acceleration of the universe deduced from the study of type Ia supernovae [14], scalar-tensor gravity has been used many times [8]. In this context, it is suggested [15] that the universe may evolve into a chaotic regime, thus making it impossible to predict its evolution in the distant future. The dimensionality of the phase space is important in this regard because a dimensional reduction of the phase space to two dimensions (which is achieved in this paper for many situations in the context of general scalar-tensor theories) is believed to inhibit the presence of chaos (see Ref. [16] for the special case of a nonminimally coupled scalar field theory). The impossibility of chaos in a two-dimensional phase space is guaranteed when the phase space is flat but it is not entirely trivial when the phase space is a curved two-dimensional surface, as is often the case in scalar-tensor cosmology [17].

In this paper we do not provide a complete phase space analysis as is done in previous works for Brans-Dicke theory and for special choices of the scalar field potential $V(\phi)$ and of the coupling function $\omega(\phi)[9]-[11]$, but we focus instead on the geometric structure and dimensionality of the phase space for any coupling function $\omega(\phi)$ and (except special cases) for any potential $V(\phi)$. A complete phase space analysis cannot obviously be performed without specifying the functions $\omega$ and $V$, but we believe that a unified treatment of the phase space geometry in the general case is of interest, especially in view of the discussion of chaotic dynamics and of the uncertainty in the form of $\omega$ and $V$. Results similar to those obtained in this paper have been presented elsewhere for a special scalar-tensor theory [18]-[22], that of a scalar field coupled nonminimally with 
the Ricci curvature and described by the action

$$
S^{(N M C)}=\int d^{4} x \sqrt{-g}\left[\left(\frac{1}{2 \kappa}-\frac{\xi}{2} \phi^{2}\right) R-\frac{1}{2} g^{a b} \nabla_{a} \phi \nabla_{b} \phi-V(\phi)\right]+S^{(m)},
$$

where $\kappa=8 \pi G$ and $\xi$ is a dimensionless coupling constant (in our notations $\xi=1 / 6$ corresponds to conformal coupling). This theory is formally a scalar-tensor theory. In fact, by redefining the scalar field according to

$$
\varphi \equiv \frac{1-\kappa \xi \phi^{2}}{G}
$$

the action (1.3) is written as

$$
S^{(N M C)}=\int d^{4} x \frac{\sqrt{-g}}{16 \pi}\left[\varphi R-\frac{\omega(\varphi)}{\varphi} g^{a b} \nabla_{a} \varphi \nabla_{b} \varphi-U(\phi)\right]+S^{(m)},
$$

where

$$
\omega(\varphi)=\frac{G \varphi}{4 \xi(1-G \varphi)}
$$

and

$$
U(\varphi)=16 \pi V[\phi(\varphi)]=16 \pi V\left( \pm \sqrt{\frac{1-G \varphi}{8 \pi G \xi}}\right) .
$$

The theories in which the geometry and the dimensionality of the phase space of scalartensor FLRW cosmology are known are summarized in Table 1. This table summarizes also the main results of the present paper, which are derived in the following sections.

\section{The equations of scalar-tensor cosmology}

In this section we consider scalar-tensor gravity as described by the action (1.2) and we write the field equations in a form that is convenient for later use. The field equations obtained by varying the action (1.2) are

$$
\begin{gathered}
G_{a b}=\frac{\omega(\phi)}{\phi^{2}}\left[\nabla_{a} \phi \nabla_{b} \phi-\frac{1}{2} g_{a b} \nabla^{c} \phi \nabla_{c} \phi\right]+\frac{1}{\phi}\left(\nabla_{a} \nabla_{b} \phi-g_{a b} \square \phi\right)-\frac{V}{2 \phi} g_{a b}+\frac{8 \pi}{\phi} T_{a b}^{(m)} \\
\square \phi=\frac{1}{2 \omega+3}\left(\phi \frac{d V}{d \phi}-2 V-\frac{d \omega}{d \phi} \nabla^{c} \phi \nabla_{c} \phi+8 \pi T^{(m)}\right)
\end{gathered}
$$


where $T_{a b}^{(m)}$ is the stress-energy tensor of ordinary matter (usually treated as a fluid) which is covariantly conserved, $\nabla^{b} T_{a b}^{(m)}=0$, and $T=T_{a}^{a}$. Note that the combination $\phi d V / d \phi-2 V$ in eq. (2.2) disappears if the potential consists of a pure mass term $V(\phi)=$ $m^{2} \phi^{2} / 2$ (which is the special case considered in Ref. [10] for Brans-Dicke theory). In the FLRW metric (1.1) the field equations assume the form

$$
\begin{gathered}
H^{2}=-H\left(\frac{\dot{\phi}}{\phi}\right)+\frac{\omega(\phi)}{6}\left(\frac{\dot{\phi}}{\phi}\right)^{2}+\frac{V(\phi)}{6 \phi}-\frac{K}{a^{2}}+\frac{8 \pi \rho^{(m)}}{3 \phi}, \\
\dot{H}=-\frac{\omega(\phi)}{2}\left(\frac{\dot{\phi}}{\phi}\right)^{2}+2 H\left(\frac{\dot{\phi}}{\phi}\right)+\frac{1}{2(2 \omega+3) \phi}\left[\phi \frac{d V}{d \phi}-2 V+\frac{d \omega}{d \phi}(\dot{\phi})^{2}\right] \\
+\frac{K}{a^{2}}-\frac{8 \pi}{(2 \omega+3) \phi}\left[(\omega+2) \rho^{(m)}+\omega P^{(m)}\right], \\
\ddot{\phi}+\left(3 H+\frac{1}{2 \omega+3} \frac{d \omega}{d \phi}\right) \dot{\phi}=\frac{1}{2 \omega+3}\left[2 V-\phi \frac{d V}{d \phi}+8 \pi\left(\rho^{(m)}-3 P^{(m)}\right)\right]
\end{gathered}
$$

where an overdot denotes differentiation with respect to the comoving time $t, H \equiv \dot{a} / a$ is the Hubble parameter, and $\rho^{(m)}$ and $P^{(m)}$ are the energy density and pressure of the material fluid, respectively. The latter satisfies the conservation equation

$$
\dot{\rho}^{(m)}+3 H\left(\rho^{(m)}+P^{(m)}\right)=0 .
$$

We assume that the matter fluid has equation of state

$$
P^{(m)}=(\gamma-1) \rho^{(m)}
$$

with $\gamma$ a constant. Then eq. (2.6) is immediately integrated to yield

$$
\rho^{(m)}=\frac{\rho_{0}}{a^{3 \gamma}}
$$

(with $\rho_{0}$ a constant), a fact that is used in the following sections. In the general case, the dynamical variables are $a$ and $\phi$, the dynamical equations (2.3) and (2.5) are of second order in $a$ and $\phi$ (there are only two independent equations in the set (2.3)-(2.5)), and the Hamiltonian constraint reduces the dimensionality of the phase space $(a, \dot{a}, \phi, \dot{\phi})$ to three, as noted in Ref. [10] for the special case of Brans-Dicke theory. In many situations 
it is possible to perform a further dimensional reduction of the phase space. First one notes that in vacuum and for $K=0$ the scale factor $a$ only appears in eqs. (2.3)-(2.5) through the combination $H \equiv \dot{a} / a$. One can then use the variables $H$ and $\phi$ and the Hamiltonian constraint to reduce the dimensionality of the phase space $(H, \phi, \dot{\phi})$ to two. It is appropriate to use the Hubble parameter because it is one of the cosmological observables.

It is sometimes useful to rewrite eqs. (2.3)-(2.5) using the conformal time $\eta$ defined by $d t=a d \eta$ and the new variable

$$
x \equiv \frac{a^{\prime}}{a}=a H
$$

where a prime denotes differentiation with respect to $\eta$. In terms of $x$ and $\eta$ one has

$$
\begin{aligned}
x^{2}= & -x\left(\frac{\phi^{\prime}}{\phi}\right)+\frac{\omega(\phi)}{6}\left(\frac{\phi^{\prime}}{\phi}\right)^{2}+\frac{a^{2} V(\phi)}{6 \phi}-K+\frac{8 \pi \rho_{0}}{3 a^{3 \gamma-2} \phi} \\
x^{\prime}= & x^{2}-\frac{\omega}{2}\left(\frac{\phi^{\prime}}{\phi}\right)^{2}+2 x\left(\frac{\phi^{\prime}}{\phi}\right)+\frac{1}{2(2 \omega+3) \phi}\left[a^{2}\left(\phi \frac{d V}{d \phi}-2 V\right)+\left(\phi^{\prime}\right)^{2} \frac{d \omega}{d \phi}\right] \\
& +K-\frac{8 \pi \rho_{0}(\omega \gamma+2)}{(2 \omega+3) \phi a^{3 \gamma-2}} .
\end{aligned}
$$

In the rest of this paper we consider various specifications of the general scalar-tensor theory (1.2) corresponding to various combinations of the scalar field potential and of the cosmic fluid.

\section{Vacuum, free Brans-Dicke-like field, and any $K$}

In the case in which no material fluid is present and $V \equiv 0$, eq. (2.10) can be written as

$$
\omega\left(\phi^{\prime}\right)^{2}-6 x \phi \phi^{\prime}-6 \phi^{2}\left(K+x^{2}\right)=0 .
$$

Provided that $\omega \neq 0$, this is a second degree algebraic equation for $\phi^{\prime}$ with reduced discriminant

$$
\frac{\Delta_{1}}{4}=3 \phi^{2}\left[(2 \omega+3) x^{2}+2 \omega K\right]
$$

and with solutions

$$
\phi_{ \pm}^{\prime}(x, \phi)=\frac{3 \phi}{\omega}\left(x \pm \sqrt{\mathcal{F}_{1}(x, \phi)}\right)
$$




$$
\mathcal{F}_{1}(x, \phi)=\frac{1}{3}\left\{[2 \omega(\phi)+3] x^{2}+2 K \omega(\phi)\right\} .
$$

If $\omega>0$ and $K=0$ or +1 it is always $\mathcal{F}_{1} \geq 0$. If $K=-1$ or $\omega<0$, there can be regions corresponding to $\mathcal{F}_{1}<0$ which are forbidden to the dynamics. The use of eq. (2.11) yields

$$
x_{ \pm}^{\prime}(x, \phi)=x^{2}+\frac{9}{2 \omega}\left(x \pm \sqrt{\mathcal{F}_{1}}\right)^{2}\left[\frac{\phi}{\omega(2 \omega+3)} \frac{d \omega}{d \phi}-1\right]+\frac{6 x}{\omega}\left(x \pm \sqrt{\mathcal{F}_{1}}\right)+K .
$$

$x^{\prime}$ and $\phi^{\prime}$ are determined once the values of $x$ and $\phi$ are given. In general there are two values of both $x^{\prime}$ and $\phi^{\prime}$ for a given pair $(x, \phi)$, corresponding to the upper and lower sign in eqs. (3.5) and (3.3), respectively. This property corresponds to the geometry of the phase space which is a two-dimensional surface embedded in the $\left(x, x^{\prime}, \phi, \phi^{\prime}\right)$ space and consists of two sheets, each of which corresponds, respectively, to the upper or lower sign in eqs. (3.3) and (3.5) - we can call them "upper sheet" and "lower sheet". The $(x, \phi)$ plane is not the phase space but is a projection of it. As a consequence, the study of the $(x, \phi)$ plane can show projections of the orbits intersecting each other. Of course the true orbits living in the curved energy surface do not cross, due to the uniqueness of the solutions of the Cauchy problem for the system (2.3)-(2.5), but projections onto the $(x, \phi)$ plane of two different orbits located in the two different sheets can intersect.

The orbits of the solutions can only change sheet (and they don't necessarily change in all the possible scalar-tensor scenarios) at points where the two sheets touch each other, i.e., where $\mathcal{F}_{1}=0$. This set of points forms the boundary of the forbidden region

$$
\mathcal{B}=\left\{\left(x, \phi, \phi^{\prime}\right): \mathcal{F}_{1}(x, \phi)=0\right\} .
$$

On points of $\mathcal{B}$ one has $x_{+}^{\prime}=x_{-}^{\prime}$ and $\phi_{+}^{\prime}=\phi_{-}^{\prime}=3 \phi x / \omega$. It should be noted that the phase space reduces to a plane instead of a curved two-surface when $\mathcal{F}_{1}$ vanishes identically, i.e., if $K=0$ and $\omega=-3 / 2$.

In the case of vacuum, $V \equiv 0$, and $K=0$ the dimensional reduction proceeds by using comoving time and the variables $H$ and $\phi$ - see Sec. 4 for an example. However if $K= \pm 1$ and comoving time is used, the scale factor cannot be eliminated from the field equations and the dimensional reduction cannot be achieved. To this purpose one must use conformal time when $K= \pm 1$. One notes that the potential can be neglected $(V \simeq 0)$ when the kinetic terms dominate the dynamics. In this case the evolution takes the universe away from the possibility of chaos and toward a two-dimensional phase space. If $V(\phi)$ dominates over the kinetic terms at late times, as in dark energy models, there is in principle the possibility of chaotic evolution, although this is not by all means guaranteed. 
The fixed points of the system (3.3) and (3.5) correspond to $\left(x^{\prime}, \phi^{\prime}\right)=(0,0)$; eqs. (3.3)(3.5) then yield either $\phi=0$, which corresponds to infinite gravitational coupling and is unphysical, or $x=0$ and $\omega K=0$. Hence,

- For $K=0$ the only fixed point is the Minkowski space corresponding to $x=a H=$ 0 , which lies on the boundary $\mathcal{B}$ of the region forbidden to the dynamics.

- For $K= \pm 1$ the only fixed points are of the form $(x, \phi)=\left(0, \phi_{0}\right)$ where $\phi_{0}$ is a root of the equation $\omega(\phi)=0$. Such a fixed point corresponds again to a Minkowski space lying on $\mathcal{B}$. If $\omega \neq 0$ everywhere there are no fixed points and, as a consequence, there are no limit cycles (a cycle must contain at least a fixed point).

\section{Vacuum, $V=m^{2} \phi^{2} / 2$, and $K=0$}

In the situation of vacuum, a massive scalar $\phi$, and $K=0$ one can use again the physical variables $H$ and $\phi$. Eq. (2.3) is rearranged as the algebraic equation for $\dot{\phi}$

$$
\frac{\omega}{6}(\dot{\phi})^{2}-H \phi \dot{\phi}+\left(\frac{m^{2}}{12} \phi-H^{2}\right) \phi^{2}=0
$$

with discriminant

$$
\Delta_{2}(H, \phi)=\frac{\phi^{2}}{3}\left[(2 \omega+3) H^{2}-\frac{\omega m^{2}}{6} \phi\right]
$$

and solutions

$$
\dot{\phi}_{ \pm}(H, \phi)=\frac{3}{\omega}\left(H \phi \pm \sqrt{\Delta_{2}}\right)
$$

The phase space is again a curved two-dimensional surface embedded in the space $(H, \phi, \dot{\phi})$ and composed of two sheets. The two sheets join on the boundary of the forbidden region described by $\Delta_{2}=0$, which is equivalent to either $\phi=0$ (unphysical) or

$$
H(\phi)= \pm m \sqrt{\frac{\phi \omega(\phi)}{6[2 \omega(\phi)+3]}} .
$$

The fixed points $\left(H_{0}, \phi_{0}\right)$ are subject to the only restriction $H_{0}= \pm m \sqrt{\phi_{0} / 12}$ and are de Sitter spaces with constant scalar field $\phi_{0} \geq 0$ located away from the boundary $\Delta_{2}=0$. Again, if $K \neq 0$ the scale factor cannot be eliminated and one cannot choose $H$ as dynamical variable. For any value of $K$ the wave equation for $\phi$ can be integrated 
yielding either the trivial solution $\phi=$ constant (corresponding to general relativity with a cosmological constant) or

$$
\int d \phi \sqrt{2 \omega(\phi)+3}=\text { const. } \int \frac{d t}{a^{3}} .
$$

A complete description of the phase space is given by Santos and Gregory [10] for BransDicke theory with $\omega=$ constant. They use the dynamical variables

$$
\begin{aligned}
X & \equiv \sqrt{\frac{2 \omega+3}{12}} \frac{\phi^{\prime}}{\phi}, \\
Y & \equiv \frac{a^{\prime}}{a}+\frac{\phi^{\prime}}{2 \phi},
\end{aligned}
$$

and conformal time $\eta$. The only difference between the Brans-Dicke case and more general scalar-tensor theories are the terms in $\dot{\omega}$ in the field equations of general scalartensor gravity. However, the dimensional reduction of the phase space cannot be achieved by using the variables $X, Y$, and $\eta$.

\section{The case of vacuum, $K=0$, and $V \neq 0$}

Let us consider again vacuum and a general self-interaction potential $V(\phi)$ for the BransDicke-like field $\phi$, but let us impose the restriction that the universe has flat spatial sections $(K=0)$. This is the situation indicated by the Boomerang [23] and MAXIMA [24] experiments for our universe. In this section we use the physically transparent variables $H$ and $\phi$ and comoving instead of conformal time. The dynamical equations reduce to

$$
\begin{gathered}
H^{2}=-H\left(\frac{\dot{\phi}}{\phi}\right)+\frac{\omega(\phi)}{6}\left(\frac{\dot{\phi}}{\phi}\right)^{2}+\frac{V(\phi)}{6 \phi}, \\
\dot{H}=-\frac{\omega(\phi)}{2}\left(\frac{\dot{\phi}}{\phi}\right)^{2}+2 H\left(\frac{\dot{\phi}}{\phi}\right)+\frac{1}{2(2 \omega+3) \phi}\left[\phi \frac{d V}{d \phi}-2 V+\frac{d \omega}{d \phi}(\dot{\phi})^{2}\right], \\
\ddot{\phi}+\left(3 H+\frac{1}{2 \omega+3} \frac{d \omega}{d \phi}\right) \dot{\phi}=\frac{1}{2 \omega+3}\left(2 V-\phi \frac{d V}{d \phi}\right) .
\end{gathered}
$$


When $\omega \neq 0$ eq. (5.1) can be rearranged as the algebraic equation for $\dot{\phi}$

$$
\omega(\dot{\phi})^{2}-6 H \phi \dot{\phi}+\left(V-6 H^{2} \phi\right) \phi=0
$$

with reduced discriminant

$$
\mathcal{F}_{2}(H, \phi)=\left[3(2 \omega+3) H^{2} \phi-\omega V\right] \phi
$$

and solutions

$$
\dot{\phi}_{ \pm}(H, \phi)=\frac{1}{\omega(\phi)}\left[3 H \phi \pm \mathcal{F}_{2}^{1 / 2}(H, \phi)\right] .
$$

The phase space is again a two-dimensional surface composed of two sheets corresponding to the lower and upper signs in eq. (5.6). Figures 1-9 illustrate the two-sheeted structure of the phase space for particular choices of $\omega(\phi)$ and $V(\phi)$. In general there is no guarantee that $\mathcal{F}_{2} \geq 0$ and there will be regions of the space $(H, \phi, \dot{\phi})$ corresponding to $\mathcal{F}_{2}<0$ forbidden to the orbits of the solutions. If $K \neq 0$ the reduction of the phase space to two dimensions cannot be performed because the scale factor appears in the expression $K / a^{2}$ in eqs. (2.3) and (2.4) and not in the form $H=\dot{a} / a$.

The phase space is flat if $\mathcal{F}_{2}=0$, i.e., if $\omega=-3 / 2$ and $V \equiv 0$. In this case one has

$$
\dot{\phi}+2 H \phi=0
$$

and either $\phi$ is identically zero (a physically unacceptable solution) or $\phi \propto a^{-2}$. Then the Hamiltonian constraint is automatically satisfied and $\dot{H}=-H^{2}$, which yields the coasting universe with scale factor $a=a_{0}\left(t-t_{0}\right)$.

In the general situation with $V \neq 0$ studied in this section, the equilibrium points correspond to $(H, \phi)=\left(H_{0}, \phi_{0}\right)$ with $H_{0}$ and $\phi_{0}$ constants and must satisfy

$$
H_{0}= \pm \sqrt{\frac{V_{0}}{6 \phi_{0}}}, \quad \phi_{0} V_{0}^{\prime}-2 V_{0}=0,
$$

where $V_{0} \equiv V\left(\phi_{0}\right)$ and $V_{0}^{\prime}=d V /\left.d \phi\right|_{\phi_{0}}$.

\section{The case $\gamma=2 / 3$ and $V \equiv 0$}

Another special case corresponds to the choice $\gamma=2 / 3$ (or $P=-\rho / 3$ ) and $V \equiv 0$. In terms of the variables $x$ and $\phi$ and of conformal time the field equations reduce to

$$
x^{2}=-x\left(\frac{\phi^{\prime}}{\phi}\right)+\frac{\omega(\phi)}{6}\left(\frac{\phi^{\prime}}{\phi}\right)^{2}-K+\frac{8 \pi \rho_{0}}{3 \phi},
$$




$$
x^{\prime}=x^{2}-\frac{\omega(\phi)}{2}\left(\frac{\phi^{\prime}}{\phi}\right)^{2}+2 x \frac{\phi^{\prime}}{\phi}+K+\frac{\left(\phi^{\prime}\right)^{2}}{2(2 \omega+3) \phi} \frac{d \omega}{d \phi}-\frac{A}{\phi},
$$

where

$$
A=\frac{16 \pi \rho_{0}(\omega+3)}{3(2 \omega+3)} .
$$

Eq. (6.1) can be rewritten as the algebraic equation for $\phi^{\prime}$

$$
\frac{\omega}{6}\left(\phi^{\prime}\right)^{2}-x \phi \phi^{\prime}+\left(\frac{8 \pi \rho_{0} \phi}{3}-K \phi^{2}-x^{2} \phi^{2}\right)=0
$$

with discriminant

$$
\Delta_{3}(x, \phi)=\frac{\phi}{3}\left[(2 \omega+3) x^{2} \phi-2 \omega\left(\frac{8 \pi \rho_{0}}{3}-K \phi\right)\right]
$$

and solutions

$$
\phi_{ \pm}^{\prime}(x, \phi)=\frac{3}{\omega}\left(x \phi \pm \sqrt{\Delta_{3}(x, \phi)}\right)
$$

while eq. (6.2) yields

$$
\begin{aligned}
x^{\prime} & =x^{2}-\frac{9}{2 \omega \phi^{2}}\left(x \phi \pm \sqrt{\Delta_{3}}\right)^{2}+\frac{6 x}{\omega \phi}\left(x \phi \pm \sqrt{\Delta_{3}}\right)+K \\
& +\frac{9}{2 \omega^{2}(2 \omega+3) \phi} \frac{d \omega}{d \phi}\left(x \phi \pm \sqrt{\Delta_{3}}\right)^{2}-\frac{A}{\phi} .
\end{aligned}
$$

The geometry of the phase space is again that of two sheets attached along the boundary $\Delta_{3}(x, \phi)=0$ of a forbidden region and embedded in the space $\left(x, \phi, \phi^{\prime}\right)$. There are no fixed points in this case. In fact, the fixed points would correspond to constant $x_{0}$ and $\phi_{0}$ and it is easy to see that eqs. (6.1) and (6.2) are incompatible when $x$ and $\phi$ are both constant.

\section{Discussion and conclusions}

The geometry and dimensionality of the phase space of FLRW scalar-tensor cosmology, in which gravity is described by the action (1.2), can be quite complicated. In general one has two independent equations for scalar-tensor cosmology, the wave equation (2.5) for the scalar $\phi$ and eq. (2.4) for the scale factor $a$. Since these are second order equa-

tions one is naturally lead to consider the phase space $(a, \dot{a}, \phi, \dot{\phi})$. The Hamiltonian 
constraint (2.3) provides a first integral and the orbits of the solutions lie on a hypersurface in this space. Hence in general the phase space is a three-dimensional curved energy hypersurface embedded in the four-dimensional space $(a, \dot{a}, \phi, \dot{\phi})$. When the spatial sections are flat $(K=0)$ the scale factor only enters the field equations through the Hubble parameter and it is convenient to choose as physical variables $H$ and $\phi$ instead of $a$ and $\phi$. The Hamiltonian constraint (2.3) then forces the traiectories of the solutions to unfold in a two-dimensional hypersurface embedded in the three-dimensional space $(H, \phi, \dot{\phi})$. However, there are also situations in which the universe has curved spatial sections $(K= \pm 1)$ and one can again reduce the phase space to a two-dimensional surface embedded in a three-dimensional space. Table 1 summarizes the situations known in the literature and the results of the present paper. The latter are derived without specifying the coupling function $\omega(\phi)$ and, in some cases, also for arbitrary scalar field potential $V(\phi)$. The stationary points of the dynamical system are determined in this general situation.

There is a simplification in the equations of scalar-tensor cosmology when the potential reduces to a mass term - previous works $[9,10]$ have taken advantage of this property. When a general scalar-tensor theory is considered instead of Brans-Dicke theory the time-dependence of $\omega(\phi)$ makes the dynamics more interesting even for vacuum, $K=0$ and a free scalar field. Even when the phase space is two-dimensional its geometry is usually non-trivial. In general the phase space accessible to the trajectories of the solutions is composed of two sheets attached to each other along the boundary $\mathcal{B}$ of a region forbidden to the dynamics. The orbits of the solutions can change sheet only by passing through points of $\mathcal{B}$. There are however scenarios in which the orbits are forced to stay in one sheet or the other but cannot change sheet - the sheet in which they live is decided by the initial conditions. The plane $(H, \phi)$ (or $(x, \phi))$ is only a projection of the true curved phase space and projections of the orbits can intersect in this plane (apart from the degenerate cases in which this plane reduces to the true phase space). In all the situations considered in this paper the fixed points of the dynamical system (when they exist) are found to be de Sitter spaces possibly degenerating into Minkowski spaces. Their stability is studied in Ref. [25].

A detailed discussion of the dynamics requires the specification of the functions $\omega(\phi)$ and $V(\phi)$. Although many forms of $V(\phi)$ have been considered in the literature on high energy physics and cosmology, there are very few suggestions about the form of the coupling function $\omega(\phi)$, and they are usually dictated by purely mathematical considerations such as the ease of finding exact cosmological solutions (cf. Ref. [4]). The clarifications on the dimensionality and geometry of the phase space made here will be useful in detailed studies fixing the form of $\omega(\phi)$ and $V(\phi)$. 


\section{Acknowledgments}

It is a pleasure to thank Prof. Werner Israel for the hospitality at the University of Victoria, where this paper was completed. This work was supported by the Natural Sciences and Engineering Research Council of Canada (NSERC).

\section{References}

[1] C.H. Brans and R.H. Dicke, Phys. Rev. 124 (1961) 925.

[2] P.G. Bergmann, Int. J. Theor. Phys. 1 (1968) 25; R.V. Wagoner, Phys. Rev. D 1 (1970) 3209; K. Nordvedt, Astrophys. J. 161 (1970) 1059.

[3] C.G. Callan, D. Friedan, E.J. Martinec and M.J. Perry, Nucl. Phys. B 262 (1985) 593; E.S. Fradkin and A.A. Tseytlin, Nucl. Phys. B 261 (1985) 1.

[4] V. Faraoni, Cosmology in Scalar-Tensor Gravity, Kluwer Academic, Dordrecht, 2004.

[5] Y. Fujii and K. Maeda, The Scalar-Tensor Theory of Gravity, Cambridge University Press, Cambridge, 2003.

[6] D. La and P.J. Steinhardt, Phys. Rev. Lett. 62 (1989) 376; E.J. Weinberg, Phys. Rev. D 40 (1989) 3950; D. La, P.J. Steinhardt and E. Bertschinger, Phys. Lett. B 231 (1989) 231; A.R. Liddle and D. Wands, Mon. Not. Roy. Astr. Soc. 253 (1991) 637.

[7] F.S. Accetta and J.J. Trester, Phys. Rev. D 39 (1989) 2854; D. La, P.J. Steinhardt and E. Bertschinger, Phys. Lett. B 231 (1989) 231; R. Holman, E.W. Kolb and Y. Wang, Phys. Rev. Lett. 65 (1990) 17; R. Holman, E.W. Kolb, S. Vadas and Y. Wang, Phys. Lett. B 269 (1991) 252; A.M. Laycock and A.R. Liddle, Phys. Rev. D 49 (1994) 1827; J. McDonald, Phys. Rev. D 48 (1993) 2462.

[8] T. Chiba, Phys. Rev. D 60 (1999) 083508; J.-P. Uzan, Phys. Rev. D 59 (1999) 123510; F. Perrotta, C. Baccigalupi and S. Matarrese, Phys. Rev. D 61 (2000) 023507; L. Amendola, Mon. Not. R. Astr. Soc. 312 (2000), 521; R. de Ritis, A.A. Marino and P. Scudellaro, Phys. Rev. D 62 (2000) 043506; X. Chen, R.J. Scherrer and G. Steigman, Phys. Rev. D 63 (2001) 123504; G. Esposito-Farése, gr-qc/0011115; A.A. Sen and R.T. Seshadri, Int. J. Mod. Phys. D 12 (2000) 445; C. Baccigalupi, S. Matarrese and F. Perrotta, Phys. Rev. D 62 (2000) 123510; Y. Fujii, Gravit. Cosmol. 6 (2000) 107; Y. Fujii and T. Nishioka, Phys. Rev. D 62 (2000) 044011; O. Bertolami and J. Martins, Phys. Rev. D 61 (2000) 064007; L. Amendola, Phys. Rev. D 62 (2000) 043511; Phys. Rev. Lett. 86 (2001) 196; B. Boisseau, G. Esposito-Farése, D. Polarski and A.A. Starobinski, Phys. Rev. Lett. 85 
(2000) 2236; M.C. Bento, O. Bertolami and N.C. Santos, Phys. Rev. D 65 (2002) 067301; T. Chiba, Phys. Rev. D 64 (2001) 103503; A. Riazuelo and J.-P. Uzan, Phys. Rev. D 62 (2000) 083506; G. Esposito-Farése and D.Polarski, Phys. Rev. D 63 (2001) 063504; N. Banerjee and D. Pavon, Class. Quant. Grav. 18 (2001) 593; V. Faraoni, Int. J. Mod. Phys. D 11 (2002) 471; D.F. Torres, Phys. Rev. D 66 (2002) 043522; F. Perrotta and C. Baccigalupi, astro-ph/0205217; A. Riazuelo and J.-P. Uzan, Phys. Rev. D 66 (2002) 023535; S. Nojiri and S.D. Odintsov, Phys. Rev. D 68 (2003) 123512; hep-th/04050078; E. Elizalde et al., Phys. Lett. B 574 (2003) 1; E. Elizalde, S. Nojiri and S.D. Odintsov, hep-th/0405034; S. Nojiiri and S.D. Odintsov, Phys. Lett. B 595 (2004) 1; S. Matarrese, C. Baccigalupi and F. Perrotta, astro-ph/0403480; R. Catena et al., astro-ph/0406152; F.C. Carvalho and A. Saa, astro-ph/0408013.

[9] S.J. Kolitch and D.M. Eardley, Ann. Phys. (NY) 241 (1995) 128; S.J. Kolitch, Ann. Phys. (NY) 246 (1996) 121.

[10] C. Santos and R. Gregory, Ann. Phys. (NY) 258 (1997) 111.

[11] D.J. Holden and D. Wands, Class. Quant. Grav. 15 (1998) 3271.

[12] R.M. Wald, General Relativity, Chicago University Press, Chicago, 1984.

[13] H. Nariai, Prog. Theor. Phys. 47 (1971) 1824; ibidem 48 (1972) 763; P. Chauvet and J.L. Cervantes-Cota, Phys. Rev. D 52 (1995) 3416; J.P. Mimoso and D. Wands, Phys. Rev. D 51 (1995) 477; A.A. Coley, J. Ibanez and R.J. van der Hoogen, Int. J. Mod. Phys. D 38 (1997) 5256; A. Billyard, A.A. Coley and J. Ibanez, Phys. Rev. D 59 (1999) 023507.

[14] A.G. Riess et al., Astron. Astrophys. 116 (1998) 1009; S. Perlmutter et al., Nature 391 (1998) 51; A.G. Riess et al., Astron. J. 118 (1999) 2668; S. Perlmutter et al., Astrophys. J. 517 (1999) 565; A.G. Riess et al., Astrophys. J. 536 (2000); A.G. Riess et al., Astrophys. J. 560 (2001) 49; A.G. Riess et al., Astrophys. J. 607 (2004) 665.

[15] A.A. Starobinsky, Gravit. Cosmol. 6 (2000) 157.

[16] E. Gunzig et al., Mod. Phys. Lett. A 15 (2000) 1363.

[17] V. Faraoni, work in progress.

[18] S. Foster, Class. Quant. Grav. 15 (1998) 3485.

[19] L. Amendola, M. Litterio and F. Occhionero, Int. J. Mod. Phys. A 5 (1990) 3861.

[20] E. Gunzig et al., Class. Quant. Grav. 17 (2000) 1783.

[21] E. Gunzig et al., Phys. Rev. D 63 (2001) 067301. 
[22] A. Saa et al., Int. J. Theor. Phys. 40 (2001) 2295.

[23] A.D. Miller et al., Astrophys. J. Lett. 524 (1999) L1; P. de Bernardis et al., Nature 400 (2000) 955; A. E. Lange et al., Phys. Rev. D 63 (2001) 042001; A. Melchiorri et al., Astrophys. J. Lett. 536 (2000) L63.

[24] S. Hanany et al., Astrophys. J. Lett. 545 (2000) L5.

[25] V. Faraoni, Phys. Rev. D 70 (2004) 044037; Phys. Rev. D 69 (2004) 123520. 


\section{Table caption}

Table 1: Situations in which the geometry and dimension of the phase space are known $(\mathrm{BD} \equiv$ Brans-Dicke theory, NMC $\equiv$ nonminimally coupled scalar field theory).

\section{Figure captions}

Figure 1: The upper sheet of the phase space of Brans-Dicke theory described by $\omega=10$ and scalar field potential $V(\phi)=m^{2} \phi^{2} / 2$ (for unit mass $m$ ).

Figure 2: The lower sheet corresponding to the situation of fig. 1.

Figure 3: The phase space of Brans-Dicke theory with massive scalar resulting from the two sheets of fig. 1 and fig. 2 glued together. The region forbidden to the dynamics appears as a hole that cannot be penetrated by the orbits of the solutions.

Figure 4: The upper sheet of the phase space of the scalar-tensor theory described by $\omega=|\phi-1|^{-1}$ and scalar field potential $V(\phi)=m^{2} \phi^{2} / 2$ (for unit mass $m$ ).

Figure 5: The lower sheet corresponding to the situation of fig. 4.

Figure 6: The phase space resulting from the two sheets of fig. 4 and fig. 5 glued together. There is a forbidden region and the two sheets touch each other only at the boundary of this region.

Figure 7: The upper sheet of the phase space of the scalar-tensor theory described by $\omega=(\phi-1)^{-4}$ and scalar field potential $V(\phi)=V_{0} \cos (\phi / m)$ (for $m$ and $V_{0}$ set to unity).

Figure 8: The lower sheet corresponding to the situation of fig. 4.

Figure 9: The phase space resulting from the two sheets of fig. 4 and fig. 5 glued together. There is a forbidden region and the two sheets touch each other only at the boundary of this region. 



\begin{tabular}{|c|c|c|c|c|c|}
\hline $\begin{array}{c}\text { Scalar-tensor } \\
\text { theory }\end{array}$ & cosmic fluid & $V(\phi)$ & $\begin{array}{c}\text { curvature } \\
\text { index }\end{array}$ & reference & phase space \\
\hline \hline BD & absent & $V=\frac{m^{2} \phi^{2}}{2}$ & any $K$ & {$[10]$} & 2-D flat \\
\hline BD & $P=-\rho / 3$ & $V=\frac{m^{2} \phi^{2}}{2}$ & any $K$ & this paper & 2-D curved \\
\hline BD & $\begin{array}{c}\text { any } \\
\text { perfect fluid }\end{array}$ & $V \equiv 0$ & any $K$ & {$[9,11]$} & 2-D flat \\
\hline BD & $\begin{array}{c}\text { any } \\
\text { perfect fluid }\end{array}$ & $V=\Lambda \phi$ & $K=0$ & {$[9]$} & 2-D flat \\
\hline BD & $\begin{array}{c}\text { any } \\
\text { perfect fluid }\end{array}$ & any $V(\phi)$ & any $K$ & {$[9,10]$} & 3-D curved \\
\hline NMC & absent & any $V(\phi)$ & $K=0$ & {$[18]-[22]$} & 2-D curved \\
\hline NMC & absent & any $V(\phi)$ & $K= \pm 1$ & {$[18]-[22]$} & 3-D curved \\
\hline $\begin{array}{c}\text { Action } \\
(1.2)\end{array}$ & absent & $V \equiv 0$ & any $K$ & this paper & 2-D curved \\
\hline Action & absent & any $V(\phi)$ & $K=0$ & this paper & 2-D curved \\
\hline absent & any $V(\phi)$ & $K= \pm 1$ & this paper & 3-D curved \\
\hline
\end{tabular}


This figure "stphase2f1.png" is available in "png" format from: http://arxiv.org/ps/gr-qc/0502015v1 
This figure "stphase3f1.png" is available in "png" format from: http://arxiv.org/ps/gr-qc/0502015v1 
This figure "stphase4f1.png" is available in "png" format from: http://arxiv.org/ps/gr-qc/0502015v1 
This figure "stphase2.png" is available in "png" format from: http://arxiv.org/ps/gr-qc/0502015v1 
This figure "stphase2f2.png" is available in "png" format from: http://arxiv.org/ps/gr-qc/0502015v1 
This figure "stphase3f2.png" is available in "png" format from: http://arxiv.org/ps/gr-qc/0502015v1 
This figure "stphase4f2.png" is available in "png" format from: http://arxiv.org/ps/gr-qc/0502015v1 
This figure "stphase3.png" is available in "png" format from: http://arxiv.org/ps/gr-qc/0502015v1 
This figure "stphase4.png" is available in "png" format from: http://arxiv.org/ps/gr-qc/0502015v1 